\title{
Dynamic Changes of High-Sensitivity Troponin T Concentration During Infancy: Clinical Implications
}

\author{
Petr JEHLICKA ${ }^{1}$, Daniel RAJDL ${ }^{2}$, Eva SLADKOVA ${ }^{1}$, Aneta SYKOROVA ${ }^{1}$, Josef \\ SYKORA ${ }^{1}$
}

${ }^{1}$ Faculty of Medicine in Pilsen, Charles University in Prague, Department of Paediatrics, University Hospital in Pilsen, Pilsen, Czech Republic, ${ }^{2}$ Charles University in Prague, Faculty of Medicine in Pilsen, Department of Clinical Biochemistry and Haematology, University Hospital in Pilsen, Pilsen, Czech Republic

Received February 21, 2020

Accepted November 18, 2020

Epub Ahead of Print January 14, 2021

\section{Summary}

Cardiac troponin $\mathrm{T}$ determination plays a dominant role in diagnosis of myocardial pathologies. Despite generally accepted use of high-sensitive cardiac troponin T assays (hscTnT) and clearly defined cut-off limit in adults, the uncertainty persists in infants. The aim of this study was to assess plasmatic concentrations of hscTnT and describe sequential age-related dynamic changes of hscTnT in healthy infants and toddlers. Seventy-eight children (52 males/26 females) from Czech Republic aged 44 to 872 days (median, interquartile range 271; 126 to 486 days) were consecutively enrolled in the singlecenter, prospective observational study. Plasma concentrations of hscTnT were analyzed by the electrochemiluminescent method, age-related reference intervals were calculated using the polynominal regression model. Amongst the study population $(n=78)$, the upper limit of hscTnT concentration defined as the $99^{\text {th }}$ percentile was calculated. The $99^{\text {th }}$ percentile with $95 \%$ confidence interval at the end of $2^{\text {nd }}, 3^{\text {rd }}, 4^{\text {th }}, 5^{\text {th }}, 6^{\text {th }}$ and $7^{\text {th }}$ month of postnatal life were: 81 (40.6 to 63.6$), 61$ (36.0 to 55.3), 47 (31.9 to 48.3 ), 37 (28.1 to 42.3 ), 30 (24.7 to 37.2 ) and 25 (21.5 to 32.7 ) ng/l, respectively. Concentration of adults $99^{\text {th }}$ percentile $(14 \mathrm{ng} / \mathrm{l})$ was achieved approximately at 1 year of postnatal life. Statistically significant negative correlation of hscTnT concentration with age $(r=-0.81, p<0.001)$ was found. Significant gender differences were not found ( $p>0.07)$. The study revealed substantially increased reference intervals of hscTnT levels in infants when compared with adult population. Based on our preliminary results, the age-related interpretation of hscTnT plasmatic concentration is recommended.

\section{Key words}

High-sensitive troponin T • Infants • Myocardial damage

\section{Corresponding author}

P. Jehlicka, Charles University in Prague, Faculty of Medicine in Pilsen, Faculty Hospital, Department of Paediatrics, Alej Svobody 80, 30400 Pilsen, Czech Republic. Fax: +420 377104694 . E-mail: jehlicka@fnplzen.cz

\section{Introduction}

Implementation of high-sensitive assays for cardiac troponin $\mathrm{T}$ assessment (hsc TnT) replaced former analytical methods in recent years. Higher analytical sensitivity allows us to measure precisely very low values of cardiac troponins in healthy population and opens us new clinical usage of cardiac troponins (e.g. rapid triage algorithms for acute myocardial infarction diagnosis or prognostic role in a wide variety of populations). These advances are not properly reflected in infants, especially aged under 1 year. Based on a historical study (Adamcova et al. 1995) using a conventional assay it is known that cardiac troponin $\mathrm{T}$ concentration to be elevated in healthy newborns. Recently, this finding was confirmed using hscTnT assay in healthy full-term neonates (Jehlicka et al. 2018, Karlen et al. 2019). Only one study based on The Canadian Laboratory Initiative on Pediatric Reference Intervals (CALIPER) cohort determined $99^{\text {th }}$ percentiles for high-sensitive cardiac 
troponin T and I in infants (Bohn 2019). However, this observation was not confirmed on other populations as recruiting of very young healthy children is cumbersome. The first aim of this study was to assess plasmatic levels of hscTnT in healthy Europe-based subjects during first months of life. The second aim was to determine agerelated $99^{\text {th }}$ percentiles of hscTnT and possible differences between males and females.

\section{Patients and Methods}

Seventy eight infants and toddlers (52 males/ 26 females) from Pilsen region (Czech Republic), aged between 44 to 872 days (median, interquartile range: 271, 126-486 days) indicated for elective minor surgery procedure (herniotomy, correction of polydactyly, pes calcaneovalgus etc.) or before planned examination by magnetic resonance were recruited in the study. Exclusion criteria were a history or symptoms of infection, use of antibiotics or anti-inflammatory agents, significantly increased C-reactive protein (CRP), detectable cardiac pathology, kidney disease or renal failure, sepsis, head or chest trauma, a history of cardiac operations or cardiotoxic medication, since cardiac troponin levels may be affected in these circumstances. CRP levels were under the Limit of Detection $(<1 \mathrm{mg} / \mathrm{l})$ in $85 \%$ of participants, $99 \%$ of participants had levels lower than $8 \mathrm{mg} / \mathrm{l}$ and one participant had concentration of CRP $21 \mathrm{mg} / \mathrm{l}$ but was free of any clinical inflammatory signs. Venous blood concentration of hscTnT was measured using the electrochemiluminescent (ECLIA) method (Roche Cobas e602 immunochemical analyser). To ensure absence of possible interference on hsTnT values by hemolysis (falsely decreased values), we determined quantitative hemolytic indices by measuring absorbance at 600 and $570 \mathrm{~nm}$ with correction for absorption due to lipemia on Roche Cobas c702 analyser. According to the manufacturer datasheet, no interference by hemolysis is reported up to $1 \mathrm{~g} / 1$. Median value of hemolysis was $0.15 \mathrm{~g} / 1$ and maximum $0.5 \mathrm{~g} / \mathrm{l}$. Thus, no interference by hemolysis on hsTnT measurement was secured. Levels of CRP were assessed by the highsensitive immunoturbidimetric assay (K-ASSAY Kamiya Biomedical Company, Seattle, USA) on Roche Cobas c702 analyser.

\section{Statistical methods}

Age-related reference intervals were calculated using Medcalc software (version 19.1.3) with log- transformed values. The statistical procedure is based on a regression model that is calculated from original data; bootstrap $95 \%$ confidence interval were calculated with 5000 iterations and random number seed 978 . The exact procedure is described elsewhere (Wright 1997). Differences between male/female groups were tested by the Mann-Whitney procedure. Correlation was calculated using non-parametric Spearman method. A p-value of 0.05 or less was considered statistically significant.

\section{Ethical considerations}

This study protocol was approved by the local Ethics Committee and performed according to principles of Declaration of Helsinki. Written informed consent was obtained in advance from the parents or legal guardians of the study participants.

\section{Results}

The upper limit of hscTnT concentration defined as the $99^{\text {th }}$ percentile was calculated using polynominal regression model. The $99^{\text {th }}$ percentile with $95 \%$ confidence interval at the end of $2^{\text {nd }}, 3^{\text {rd }}, 4^{\text {th }}, 5^{\text {th }}, 6^{\text {th }}$ and $7^{\text {th }}$ month of postnatal life were: 81 (40.6 to 63.6), 61 (36.0 to 55.3), 47 (31.9 to 48.3 ), 37 (28.1 to 42.3 ), 30 (24.7 to 37.2$)$ and 25 (21.5 to 32.7$) \mathrm{ng} / \mathrm{l}$ respectively. Concentration of "adult" $99^{\text {th }}$ percentile (14 ng/l) was achieved approximately at 1 year of postnatal life. The distribution of $97.5^{\text {th }}$ and $99^{\text {th }}$ percentile of hsTnT values in the study groups is shown in Table 1. For practical purposes, simplified values of $99^{\text {th }}$ percentiles based on authors' expert opinion were derived (Table 1). There was a strong statistically significant negative correlation of hscTnT concentration with age $(\mathrm{r}=-0.81, \mathrm{p}<0.001)$. This relationship was graphically most apparent during the first 200 days of life (Fig. 1). Girls tended to have lower values of hscTnT than boys $(\mathrm{p}=0.07)$, median $(95 \%$ CI $)$ difference was $1.6 \mathrm{ng} / \mathrm{l}(-0.2$ to $5.8 \mathrm{ng} / \mathrm{l}$ (Fig. 2).

One participant was excluded due to unexpectedly high hscTnT concentration (119.5 ng/l at age 51 days).

\section{Discussion}

Our study is the first descripting age-related plasma concentrations of hscTnT in healthy children from middle Europe aged 44 to 872 days. It expands our knowledge about natural age-related changes of cardiac 
Table 1. Age-related $97.5^{\text {th }}$ and $99^{\text {th }}$ percentile with respective $95 \%$ confidence intervals $(n=78)$ grouped by 30 days and longer intervals.

\begin{tabular}{|c|c|c|c|}
\hline Age (days) & $97.5^{\text {th }}$ percentile $(95 \% \mathrm{CI})$ & $99^{\text {th }}$ percentile $(95 \% \mathrm{CI})$ & Consensual $99^{\text {th }}$ percentile (ng/l) \\
\hline 60 & $69(38.3$ to 58.8$)$ & $81(40.6$ to 63.6$)$ & 80 \\
\hline 90 & $52(33.9$ to 51.0$)$ & $61(36.0$ to 55.3$)$ & 60 \\
\hline 120 & $41(29.8$ to 44.3$)$ & 47 (31.9 to 48.3$)$ & 50 \\
\hline 150 & $32(26.3$ to 38.9$)$ & 37 (28.1 to 42.3$)$ & 40 \\
\hline 180 & $26(23.0$ to 34.2$)$ & $30(24.7$ to 37.2$)$ & 30 \\
\hline 210 & $22(20.0$ to 29.9$)$ & $25(21.5$ to 32.7$)$ & 25 \\
\hline 260 & $17(15.6$ to 23.9$)$ & $20(16.8$ to 26.1$)$ & 20 \\
\hline 360 & $12(9.7$ to 15.4$)$ & $14(10.3$ to 16.7$)$ & 14 \\
\hline 720 & $10(7.4$ to 18.2$)$ & $11(7.8$ to 20.2$)$ & 10 \\
\hline
\end{tabular}

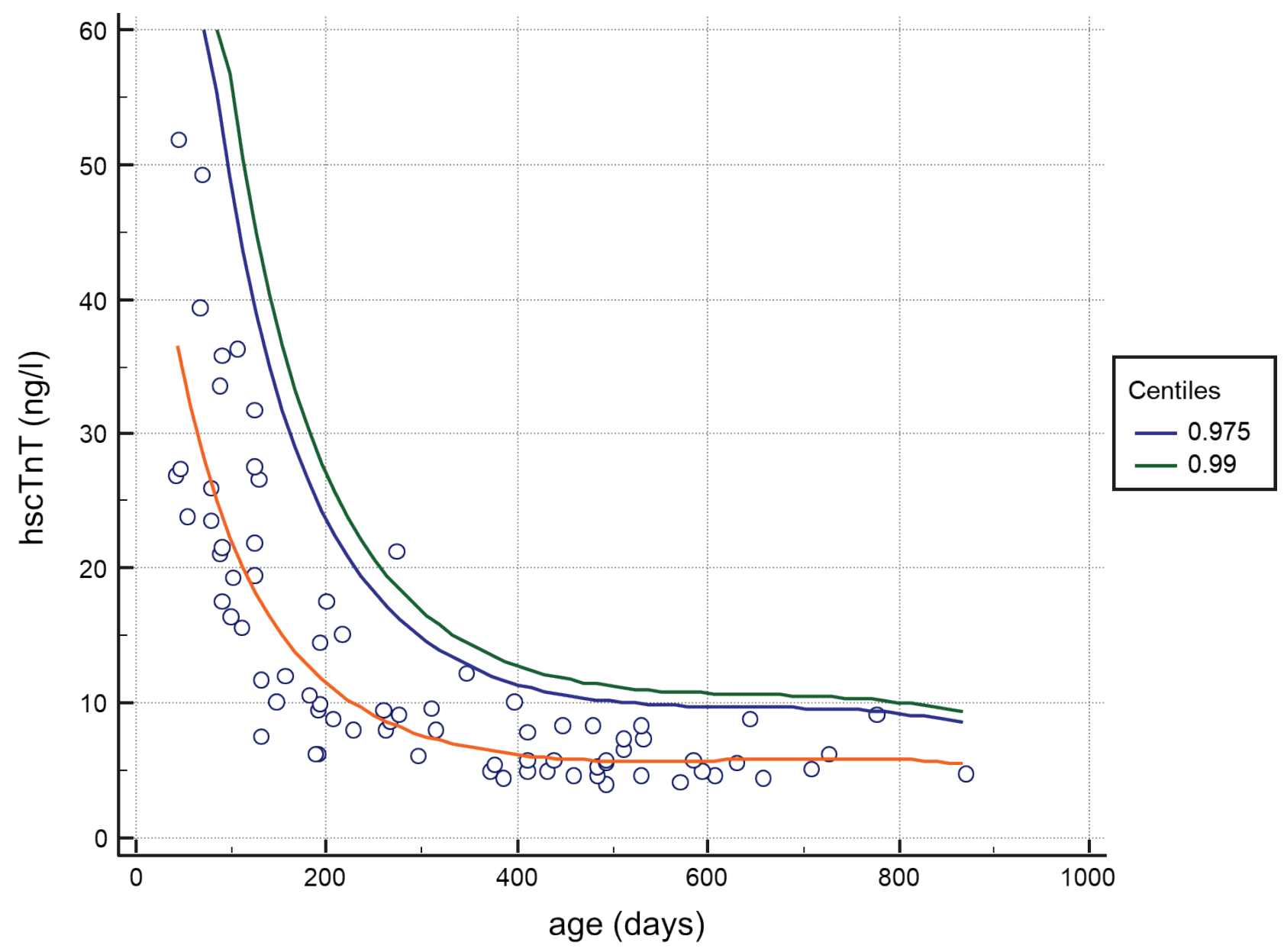

Fig. 1. Continuous Age-related $97.5^{\text {th }}$ and $99^{\text {th }}$ percentile derived from polynomial regression model. 


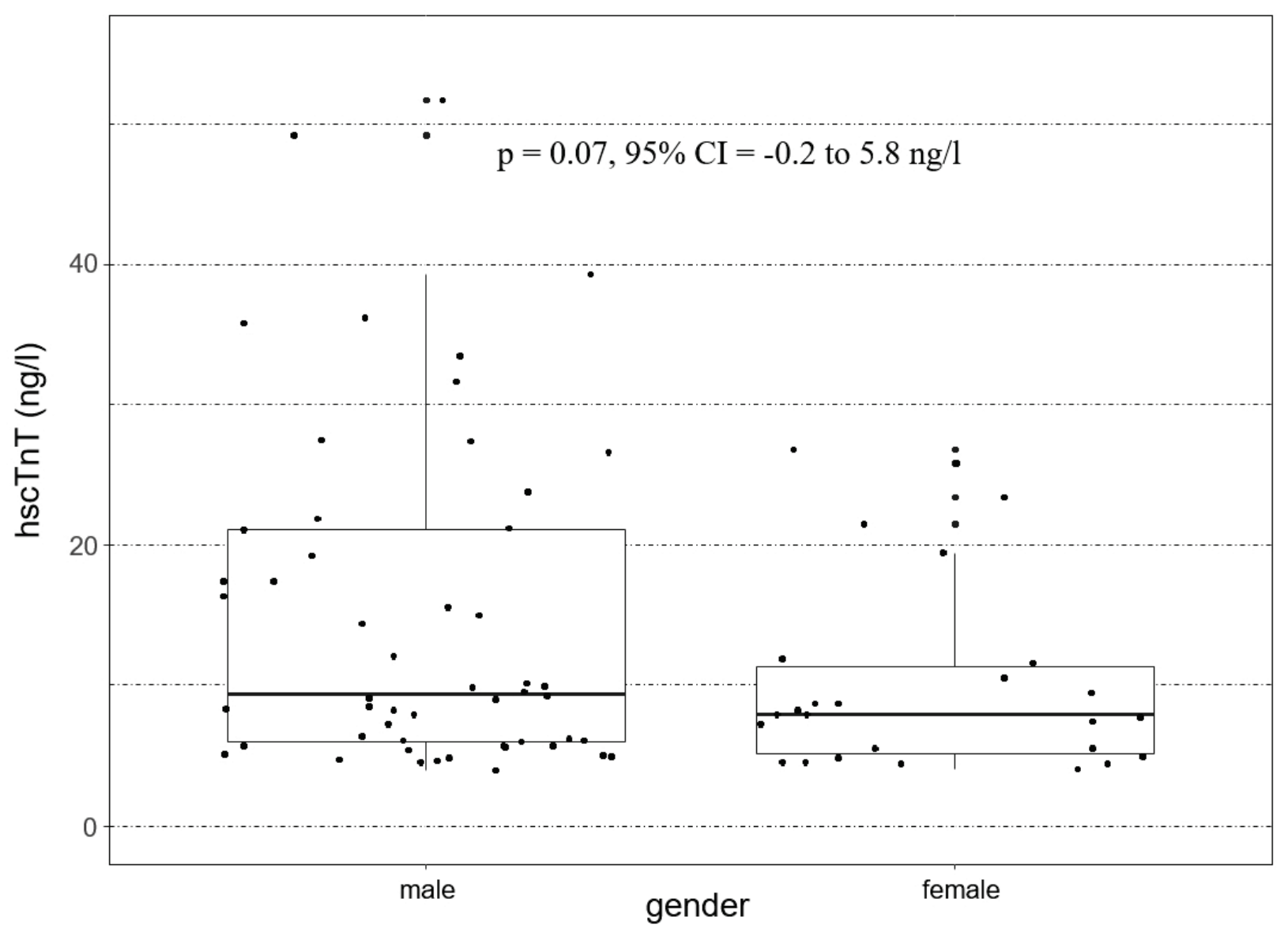

Fig. 2. Differences in plasma hscTnT values between males and females.

troponin in infants and provides us with several useful conclusions: 1) hscTnT concentrations were considerably higher in infants than in adults; 2) gradual decline of hscTnT level was described within the first year of life; after that period, plasmatic concentrations of hscTnT are comparable with adult population; 3) preliminary agerelated ranges of hscTnT were established.

As has been reported in our previous study (Jehlicka et al. 2018), the upper reference interval of hscTnT is significantly higher in neonates when compared with adult reference values. Recently published Swedish study presented similar results (Karlen 2019). This finding is in agreement with former studies using conventional troponin $\mathrm{T}$ and I assays in healthy neonates (Baum et al. 2004, Clark et al. 2001). The gradual decline of hscTnI values during the first year of life was observed (Caselli et al. 2016), nevertheless the age-related reference intervals for hsTnI were not been published. HscTnI assays are not harmonized and this fact makes it difficult to generalize results for other hsTnI assays to date. However, it is quite evident that the increase of both cardiac troponin $\mathrm{T}$ and $\mathrm{I}$ is present in the first period of life. Bohn et al. (2019) very recently published $99^{\text {th }}$ percentiles for hsTnT derived from a pediatric population (CALIPER study) that included probands up to 19 years old. This study enrolled 99 males and females under 1 year and provided with our results comparable $99^{\text {th }}$ percentiles for this population $(93 \mathrm{ng} / 1$ under 6 months of age and $19 \mathrm{ng} / \mathrm{l}$ between 6 months and 1 year of age). As the changes of hsTnT in the first 6 months of life are very turbulent, we used different statistical approach based on regression modelling that allowed us to derive more detailed estimates of $99^{\text {th }}$ percentiles. This approach brings a useful new view on interpretation of hsTnT values as there are clearly significantly higher hsTnT values in 1 month vs. 6 months.

Underlying mechanism leading to the elevation of hscTnT and its strong negative correlation with age reflects many pathophysiological mechanisms and has not been fully explained. The half-life of troponin $\mathrm{T}$ and troponin I in the blood is about $2 \mathrm{~h}$ (Gerhardt et al. 1991). After myocardial injury, the release of cardiac troponins into plasma is probably highly dependent on blood flow in the damaged tissue and the idea of "unbound" part of 
troponin I and T in the cytosol is not supported by recent publications (Katus et al. 1991, Starnberg et al. 2014). Rapid rise, peak and slower fall of cardiac troponins after acute myocardial injury is probably driven by the reperfusion of damaged tissue and sustained release of cardiac troponins from necrotic myocardium. Elevated plasma concentrations of hscTnT due to myocardial ischemia decline to normal levels in successfully treated adult patients within 2 weeks (Skeik et al. 2008). The possible influence of physical stress and transient hypoxia on myocardium during vaginal birth (Costa et al. 2007, Cruz et al. 2006) could be in accordance with studies reporting elevation of cardiac troponin $\mathrm{T}$ in healthy adults after strenuous physical exertion (Apple et al. 2009, Hewing et al. 2015). However, neither physical stress nor transient perinatal hypoxia can elucidate a substantially longer persistence of elevated hscTnT plasma concentrations in healthy infants (Fig. 1, Table 1). We assume that the shift of pressure afterload from the right to the left ventricle associated with decreasing pulmonary resistance might be linked with programmed apoptosis and cardiomyocyte proliferation during early infancy. This mechanism can lead to releasing of small quantities of troponins into the circulation that are detectable by high-sensitive assays. The hypothesis is concordant with the published study describing gradual decrease of fetal slow-skeletal troponin I and slow transition of myoglobin light chains into the adult form in very young children operated for complex congenital heart defects (Elhamine et al. 2016). The slow transformation was independent of the type of heart defect or surgery and might be of developmentally programmed origin. It is known that human cardiomyocytes proliferate and enlarge after birth (Mollova et al. 2012). We can hypothesize that small dense cardiomyocytes in the neonatal and early infant period have different metabolism and faster turnaround of troponins.

HscTnT concentrations were higher in males than females but the difference was not statistically significant. The finding is in agreement with hscTnT studies in neonates (Jehlicka et al. 2018) and infants under 1 year (Bohn et al. 2019).

\section{Strengths and weaknesses}

The strength of the study was the creating of a clearly defined cohort with consistent genetic background in a prospective manner, using of certified methodological techniques and well-defined selection criteria. These factors decreased the risk of selection bias. On the other hand, some limitations must be considered when interpreting our results. As a single-center study, the number of probands was relatively small. The main reason was the ethically problematic venous sampling in healthy infants and toddlers. The consistent pattern of measured values allowed us building a robust regression model for derivation of age-related reference intervals with reasonable estimates. We present our data as preliminary requiring confirmation in larger studies and to take a cautious approach when interpreting findings to date. Further studies are needed to define clinically sound cut-offs for different pediatric pathologies. However, we are confident that our results retain substantial relevance and several potential limitations did not compromise our extrapolations and the clinical significance of our findings.

\section{Conclusions}

Our study has revealed substantially increased reference intervals of hscTnT in infants when compared with adult population. The strong negative correlation between hscTnT and age was confirmed within the first year of life. Based on our preliminary results, the age-related interpretation of hscTnT plasma concentration is recommended in infants.

\section{Conflict of Interest}

There is no conflict of interest.

\section{Acknowledgements}

This study was conducted within research project PROGRES O-39 and the grant of Ministry of Health of the Czech Republic - Conceptual Development of Research Organization (Faculty Hospital in Pilsen FNP1, 00669806).

\section{References}

ADAMCOVA M, KOKSTEIN Z, PALICKA V, PODHOLOVA M, KOSTAL M: Troponin T levels in the cord blood of healthy term neonates. Physiol Res 44: 99-104, 1995.

APPLE FS: A new season for cardiac troponin assays: it's time to keep a scorecard. Clin Chem 55: 1303-1306, 2009. https://doi.org/10.1373/clinchem.2009.128363 
BAUM H, HINZE A, BARTES P, NEUMAIER D: Reference values for cardiac troponins T and I in healthy neonates. Clin Biochem 37: 1079-1082, 2004. https://doi.org/10.1016/j.clinbiochem.2004.08.003

BOHN MK, HIGGINS V, KAVSAK P, HOFFMAN B, ADELI K: High-sensitivity generation 5 cardiac troponin T sex- and age-specific 99th percentiles in the CALIPER cohort of healthy children and adolescents. Clin Chem 65: 589-592, 2019. https://doi.org/10.1373/clinchem.2018.299156

CASELLI C, CANGEMI G, MASOTTI S, RAGUSA R, GENNAI I, DEL RY S, PRONTERA C, CLERICO A: Plasma cardiac troponin I concentrations in healthy neonates, children and adolescents measured with a high sensitive immunoassay method: High sensitive troponin I in pediatric age. Clin Chim Acta 458: 68-71, 2016. https://doi.org/10.1016/j.cca.2016.04.029

CLARK SJ, NEWLAND P, YOXALL CW, SUBHEDAR N: Cardiac troponin T in cord blood. Arch Dis Child Fetal Neonatal Ed 84: 34-37, 2001. https://doi.org/10.1136/fn.84.1.F34

COSTA S, ZECCA E, De ROSA G, DE LUCA D, BARBATO G, PARDEO M, ROMAGNOLI C: Is serum troponin T a useful marker of myocardial damage in newborn infants with perinatal asphyxia? Acta Paediatr 96: 181-184, 2007. https://doi.org/10.1111/j.1651-2227.2007.00104.x

CRUZ MA, BREMMER YA, PORTER BO, PORTER BO, GULLQUIST SD, WATTERBERG KL, ROZYCKI HJ: Troponin T and cardiac dysfunction in extremely low-birth-weight infants. Pediatr Cardiol 27: 396-401, 2006. https://doi.org/10.1007/s00246-005-0942-3

ELHAMINE F, IORGA B, KREUGER M, HUNGER M, ECKHARDT J, SREERAM N, BENNINK G, BROCKMEIER K, PFITZER G, STEHLE R: Postnatal development of right ventricular myofibrillar biomechanics in relation to the sarcomeric protein phenotype in pediatric patients with conotruncal heart defects. J Am Heart Assoc 5: e003699, 2016. https://doi.org/10.1161/JAHA.116.003699

GERHARDT W, KATUS H, RAVKILDE J, HAMM C, JØRGENSEN PJ, PEHEIM E, LJUNGDAHL L, LÖFDAHL $\mathrm{P}$ : S-troponin $\mathrm{T}$ in suspected ischemic myocardial injury compared with mass and catalytic concentrations of S-creatine kinase isoenzyme MB: Clin Chem 37: 1405-1411, 1991. https://doi.org/10.1093/clinchem/37.8.1405

HEWING B, SCHATTKE S, SPETHMANN S, SANAD W, SCHROECKH S, SCHIMKE I, HALLECK F, PETERS H, BRECHTEL L, LOCK J BAUMANN G, DREGER H, BORGES AC, KNEBEL F: Cardiac and renal function in a large cohort of amateur marathon runners. Cardiovasc Ultrasound 13: 13, 2015. https://doi.org/10.1186/s12947-015-0007-6

JEHLICKA P, HUML M, RAJDL D, MOCKOVA A, MATAS M, DORT J, MASOPUSTOVA A: How to interpret elevated plasmatic level of high-sensitive troponin T in newborns and infants? Physiol Res 67: 191-195, 2018. https://doi.org/10.33549/10.33549/physiolres.933704

KARLEN J, KARLSSON M, ELIASSON H, BONAMY AE, HALVORSEN CP: Cardiac troponin T in healthy full-term infants. Pediatr Cardiol 40: 1645-1654, 2019. https://doi.org/10.1007/s00246-019-02199-9

KATUS H.A, REMPPIS A, SCHEFFOLD T, DIEDERICH KW, KUEBLER W: Intracellular compartmentation of cardiac troponin $\mathrm{T}$ and its release kinetics in patients with reperfused and nonreperfused myocardial infarction. Am J Cardiol 67: 1360-1367, 1991. https://doi.org/10.1016/0002-9149(91)90466-X

MOLLOVA M, BERSELL K, WALSH S, SAVLA J, DAS LT, PARK SY, SILBERSTEIN LE, DOS REMEDIOS CG, GRAHAM D, COAN S, KUHN B: Cardiomyocyte proliferation contributes to heart growth in young humans. Proc Natl Acad Sci U S A 110: 1446-1451, 2013. https://doi.org/10.1073/pnas.1214608110

SKEIK N, PATEL DC: A review of troponins in ischemic heart disease and other conditions. Int J Angio 16: 53-58, 2007. https://doi.org/10.1055/s-0031-1278248

STARNBERG K, JEPPSSON A, LINDAHL B, HAMMARSTEN O: Revision of the troponin T release mechanism from damaged human myocardium. Clin Chem 60: 1098-1104, 2014. https://doi.org/10.1373/clinchem.2013.217943

WRIGHT EM, ROYSTON P: A comparison of statistical methods for age-related reference intervals. J Royal Stat Soc Series A Stat Soc 160: 47-69, 1997. https://doi.org/10.1111/1467-985X.00045 